\title{
The Phases and Triviality of Scalar Quantum Electrodynamics
}

\author{
M. Baig and H. Fort \\ Grup de Física Tè̀rica, Institut de Física d'Altes Energies, Universitat Autònoma de Barcelona, \\ 08193 Bellaterra (Barcelona) SPAIN \\ J. B. Kogut \\ Physics Department, 1110 West Green Street, University of Illinois, Urbana, IL 61801-3080 \\ S. Kim \\ High Energy Physics Division, Argonne National Laboratory, Argonne, IL 60439
}

(April, 1994)

\begin{abstract}
The phase diagram and critical behavior of scalar quantum electrodynamics are investigated using lattice gauge theory techniques. The lattice action fixes the length of the scalar ("Higgs") field and treats the gauge field as non-compact. The phase diagram is two dimensional. No fine tuning or extrapolations are needed to study the theory's critical behovior. Two lines of second order phase transitions are discovered and the scaling laws for each are studied by finite size scaling methods on lattices ranging from $6^{4}$ through $24^{4}$. One line corresponds to monopole percolation and the other to a transition between a "Higgs" and a "Coulomb" phase, labelled by divergent specific heats. The lines of transitions cross in the interior of the phase diagram and appear to be unrelated. The monopole percolation transition has critical indices which are compatible with ordinary four dimensional percolation uneffected by interactions. Finite size scaling and histogram methods reveal
\end{abstract}


that the specific heats on the "Higgs-Coulomb" transition line are well-fit by the hypothesis that scalar quantum electrodynamics is logarithmically trivial. The logarithms are measured in both finite size scaling of the specific heat peaks as a function of volume as well as in the coupling constant dependence of the specific heats measured on fixed but large lattices. The theory is seen to be qualitatively similar to $\lambda \phi^{4}$.

The standard CRAY random number generator RANF proved to be inadequate for the $16^{4}$ lattice simulation. This failure and our "work-around" solution are briefly discussed.

11.10Gh, 11.15.Ha, 11.30.Qc

Typeset using REVTEX 


\section{INTRODUCTION}

In a recent letter ${ }^{1 .}$ we presented a lattice gauge theory study of scalar quantum electrodynamics (SQED) which provided strong numerical evidence for the logarithmic triviality of the theory. It is the purpose of this paper to both provide further detail underlying that letter, as well as present a more comprehensive view of SQED by discussing additional lattice calculations. These new calculations will include monopole percolation observables, the coupling constant dependence of the model's specific heat, evidence for logarithms of triviality in the finite size scaling variable of the model's specific heat peaks and a simulation of the four dimensional planar spin model. We shall see that there is a line of monopole percolation transitions in the phase diagram of SQED, but unlike fermionic lattice QED, it does not coincide with the bulk transition separating the Higg's and Coulomb phases of the model and is, therefore, irrelevant to the theory's continuum limit. We will investigate the theory's continuum limit for a fairly large value of the bare gauge coupling. As already reported in ref.1, the Higg's-Coulomb phase transition will prove to be compatible with a logarithmically trivial continuum theory. Finite size scaling studies of the specific heat peaks and their positions in the phase diagram as a function of lattice volume, point to logarithmically improved mean field theory as an accurate effective field theory. The correlation length exponent $\nu$ is $0.50(2)$, which is compatible with the free field result of $1 / 2$. The specific heat peaks do grow with lattice size, but the data strongly favor a slow logarithmic volume dependence rather that the power law dependence expected of a non-trivial continuum theory. New measurements of the dependence of the specific heats on the bare coupling constants also expose logarithmic modifications of pure mean field predictions. In fact, this study supports the view that SQED has scaling behavior which is qualitatively similar to $\lambda \phi^{4}$.

There are several theoretical as well as phenomenological motivations for this work. On the theory side, the search continues for an interacting ultra-violet fixed point field theory in four dimensions. Our numerical evidence suggests that SQED suffers from the zero charge problem $^{2 .}$ like $\lambda \phi^{4}$. Another theoretical motivation for this work is our recent investigation 
of fermionic QED whose simulation results could be fit with the scaling laws of a nontrivial field theory with an ultra-violet stable fixed point ${ }^{3 .}$. It was also observed in those simulations that monopole percolation is coincident with the chiral transition and that the chiral transition has the same correlation length index $\nu$ as four dimensional percolation. These two coincidences have led to the speculation that monopole percolation is "driving" a non-trivial chiral transition in lattice fermion QED and this is leading to an interacting ultra-violet fixed point. The spin $1 / 2$ character of the fermion is essential in this physical picture because a percolating network of monopoles can induce rapid helicity flips leading to chiral symmetry breaking and the index $\nu$ of the monopole network could be inherited by the chiral transition ${ }^{3 .}$. We do not expect such sensitivity to monopoles in SQED, and we shall find that the Higgs-Coulomb transition appears to be unrelated to monopole percolation since the transition lines for each phenomena are separate and actually cross in the interior of the model's two dimensional phase diagram.

This article is organized into several sections. In Sec. 2 we map out the two dimensional phase diagram of the model, and show that the Higgs-Coulomb line is separate from the monopole percolation line. The main concepts and observables of monopole percolation are briefly reviewed. In Sec. 3 we present the finite size scaling data and analysis of the specific heat peak characterizing the Higgs-Coulomb transition at a fixed, large gauge coupling. The logarithmic growth of the peak is quite clear in the data. In addition, the finite size dependence of the critical coupling is well fit with a correlation length index $\nu=.50(2)$ which is compatible with a free field description of the transition. A careful study of the Binder Cumulant and related moments of the specific heat data confirms that the transition is second order. No evidence is found for a weak or fluctuation-induced transition that bedevil other lattice studies of the Higgs model. This result is a clear advantage of the non-compact gauge/fixed length Higgs field formulation used here. Finally, we present new specific heat measurements on $12^{4}, 16^{4}$, and $20^{4}$ lattices which show further evidence for logarithmic triviality. In particular, using histogram methods, we obtained the shape of the specific heat peaks on each lattice. The resulting curves could be mapped onto a universal specific 
heat curve if scale breaking logarithms are incorporated into the otherwise gaussian model finite size scaling variable. This analysis is inspired by recent work in $\lambda \phi^{4}$ models and is, to our knowledge, the first quantitatively interesting study of its kind. In Sec. 4 we present the data and analysis of a nearby point on the monopole percolation line of transitions. The data are consistent with the scaling laws of four dimensional percolation. In fact, we find that the ratio of the monopole susceptibility index $\gamma$ to the monopole correlation length index $\nu$ is $2.25(1)$, in perfect agreement with the presumably exact result $9 / 4$. In Sec. 5 we present data and analysis of the limit of SQED where the gauge coupling is set to zero and the model reduces to the $O(2)$ spin model. In this limit mean field theory modified by calculable scale breaking logarithms should apply. The point of this exercise is not to present yet another study of the fixed length $O(2)$ model, but simply to check that the methods, lattice sizes, and statistics used in the rest of this work can reproduce known answers. Finally, in Sec. 6 we present some conclusions and suggestions for related work.

\section{PHASE DIAGRAM AND OVERVIEW}

We begin with a lattice formulation of scalar electrodynamics which is particularly well suited for numerical work and can make contact with continuum physics with a minimum of fine tuning. Consider the non-compact formulation of the abelian Higgs model with a fixed length scalar field, ${ }^{4}$.

$$
S=\frac{1}{2} \beta \sum_{p} \theta_{p}^{2}-\gamma \sum_{x, \mu}\left(\phi_{x}^{*} U_{x, \mu} \phi_{x+\mu}+\text { c.c. }\right)
$$

where $\mathrm{p}$ denotes plaquettes, $\theta_{p}$ is the circulation of the non-compact gauge field $\theta_{x, \mu}$ around a plaquette, $\beta=1 / e^{2}$ and $\phi_{x}=\exp (i \alpha(x))$ is a phase factor at each site. We choose this action (the electrodynamics of the planar model) because preliminary work has suggested that it has a line of second order transitions, ${ }^{4}$ because it does not require fine tuning and because it is believed to lie in the same universality class as the ordinary lattice abelian

Higgs model with a conventional, variable length scalar field. ${ }^{5}$ In Fig. 1 we show the phase 
diagram of the model in the bare parameter space $\beta-\gamma$. In the "Higgs" region of the phase diagram the gauge field develops a mass dynamically, while in the "Coulomb" phase it does not. Earlier work on this model indicates that the phase transition shows up clearly in the model's internal energies. A preliminary investigation has indicated that the line emanating from the $\beta \rightarrow \infty$ limit of Fig. 1 is a line of critical points which potentially could produce a family of interacting, continuum field theories. ${ }^{4}$. Note that in the $\beta \rightarrow \infty$ limit the gauge field in Eq. (1) reduces to a pure gauge transformation so the model becomes the four dimensional planar model which is known to have a second order phase transition which is trivial, i.e. is described by a free field. The $\gamma \rightarrow \infty$ limit of the transition line is also interesting and was discussed briefly in Ref. 4. The non-compact nature of the gauge field is important in Fig. 1-the compact model has a line of first order transitions and only at the endpoint of such a line in the interior of a phase diagram can one hope to have a critical point where a continuum field theory might exist. ${ }^{6}$. Since one must fine tune bare parameters to find such a point, the compact formulation of the model is much harder to use for quantitative work. ${ }^{6}$. The fact that Eq. (1) uses fixed length scalar fields avoids another fine tuning-the variable length scalar field formulation would possess a quadratically divergent bare mass parameter which would have to be tuned to zero with extraordinary accuracy to search for critical behavior. Conventional wisdom based on the renormalization group states that Eq. (1) should have the same critical behavior as the fine-tuned, variable length model, ${ }^{5}$ so it again emerges as preferable. Note also that in the naive classical limit where the field varies smoothly, Eq. (1) reduces to a free massive vector boson. In the vicinity of the strong coupling critical point we investigate here, the fields are rapidly varying on the scale of the lattice spacing and we shall see that the specific heat scaling law is not that of a Gaussian model.

In order to map out the phase diagram we first measured the internal energies,

$$
E_{\gamma}=\frac{1}{2}<\sum_{p} \theta_{p}^{2}>, \quad E_{h}=<\sum_{x, \mu} \phi_{x}^{*} U_{x, \mu} \phi_{x+\mu}+\text { c.c. }>
$$


on small lattices. This approach has been used in the past to map out the Higgs-Coulomb phase transition line in similar models ${ }^{5}$.

To locate the line of Higgs-Coulomb transitions we made "heating" and "cooling" runs along lines of fixed $\beta$ or $\gamma$ on a $6^{4}$ lattice. Typically, 250 iterations were done at each coupling and measurements were taken. Then the relevant coupling was changed by \pm 0.005, and another 250 iterations were made, etc. These hystersis runs were repeated with greater statistics in several cases. For example, the results at $\beta=0.1$ and 0.2 shown in Fig. 2 and 3 resulted from runs with 6,000 iterations per point. Both internal energies suggest a Higgs-Coulomb phase transition at $\gamma=.35$ when $\beta=0.1$, and both suggest that it moves to $\gamma=.25$ when $\beta$ is set to 0.2 . Runs of this sort were also done over a wide range of $\gamma$ and $\beta$ values, and the two dimensional contour plots of the internal energies shown in Fig. 4 and 5 resulted. The resulting line of the Higgs-Coulomb transitions is shown in Fig. 6 as the continuous, dark line. This crude map of the phase diagram will prove very helpful in guiding the large scale simulations which will be described below. We will confirm that the Higgs-Coulomb phase transition is second order and is compatible with the logarithmic triviality of SQED.

It would be interesting to understand the dynamics behind the Higgs-Coulomb phase transition in this model. The limiting case of the model when $\beta$ approaches infinity (the gauge coupling $g^{2}$ vanishing) corresponds to the four dimensional planar spin model which experiences an order-disorder transition in $\gamma$ which is described by mean field theory modified by calculable scale breaking logarithms. The major issue in this investigation is whether this transition becomes nontrivial as the gauge coupling is taken different from zero and we move inside the phase diagram of Fig. 6. Long range vector forces are certainly capable of doing this and there are many examples of similar phenomena in the statistical mechanics literature. Four dimensional model field theories, such as the gauged Nambu-Jona Lasinio model solved in the ladder approximation, have analogous behavior ${ }^{7}$ : when the gauge coupling is set to zero the model reduces to the pure Nambu-Jona Lasinio model which has a (chiral) transition which is trivial, but when the gauge coupling is nonzero the theory 
develops anomalous dimensions which grow with $g^{2}$. Of course, the gauged Nambu-Jona Lasinio model has not been solved beyond the ladder approximation so it is not known if the screening produced by internal fermion loops reduces the effective gauge coupling to zero rendering the theory noninteracting. This problem is under active research by lattice methods using the noncompact formulation of fermionic QED.

In addition to studying the usual order parameters and bulk thermodynamic quantities in order to search for phase transitions and scaling laws, it has proved stimulating to also consider the effective monopole operators introduced by Hands and Wensley ${ }^{8}$. The reader should consult the references for background on this extensive subject, so we will just review some of the essentials here. Even though the lattice action is noncompact, one can have finite action monopole loops on the lattice by virtue of the lattice cutoff. These monopoles are not necessarily physically significant because the pure gauge action is noncompact and purely gaussian. For example, effective monopoles can be found in the quenched model ${ }^{8 .}$ which is a free field and the effective monopoles cannot interact or experience real dynamics like the monopoles of pure compact lattice gauge theory. However, as emphasized in ref.8, since matter fields couple to gauge fields through phase factors which implement the $U(1)$ gauge group, they could be significant and physical in the full theory. In fact, in noncompact fermion lattice QED with two or four species, the chiral transitions are coincident with the monopole percolation transition and they share the same correlation length scaling index $\nu^{3}$. These points have led to the inevitable speculation that monopole percolation is an essential ingredient in the chiral transitions in the fermion models. These are subjects of active research and many pieces are missing in the puzzles associated with these ideas. Nonetheless, it is interesting to look for monopole percolation in SQED and see if it is related to the Higgs-Coulomb transition found in the bulk thermodynamics. In fact, we shall find that the two transitions are not coincident in the two dimensional phase diagram of SQED.

Monopole percolation is detected using an order parameter and a susceptibility borrowed from standard percolation models. In this construction a conserved magnetic current is defined on the dual lattice exactly as it is done in compact lattice QED $^{9}$. Then the idea of 
a connected cluster of monopoles is introduced: one counts the number of dual sites joined into clusters by monopole line elements. An order parameter for a percolation transition is then $M=n_{\max } / n_{t o t}$, where $n_{\max }$ is the number of such sites in the largest monopole cluster and $n_{t o t}$ is the total number of connected sites. Its associated susceptibility reads,

$$
\chi=\left\langle\left(\sum_{n} g_{n} n^{2}-n_{\max }^{2}\right) / n_{t o t}\right\rangle
$$

where $n$ labels the number of sites in a monopole cluster which occurs $g_{n}$ times on the dual lattice.

The percolation order parameter $M$ and its susceptibility $\chi$ were then calculated at fixed values of $\gamma$ and variable $\beta$ on a $10^{4}$ lattice to search for line(s) of percolation transitions. Our past experience with quenched noncompact lattice QED as well as the two and four species models suggested that the line of percolation transitions would occur near $\beta=0.2$ and be relatively insensitive to $\gamma$. The simulation gave results in good agreement with these expectations. The percolation transition line (dashed, with squares) is shown in Fig. 6. The squares in Fig. 6 denote the maxima found in the percolation susceptibility in simulation runs in which $\gamma$ was held fixed on a $10^{4}$ lattice, and "heating" and "cooling" runs were made across the peak. Typically, 4,000 iterations were made for thermalization at each $\beta$, then an additional 16,000 iterations were made for measurements. Next, $\beta$ was incremented by \pm 0.002 and the process was repeated. Accurate measurements and finite size scaling studies of $M$ and $\chi$ on larger lattices will be discussed below. The line was located from peaks in the percolation susceptibility and some examples of such measurements will be plotted in Sec. 4 where a quantitative finite size scaling study of the percolation transition will be reported.

The first thing we notice from these measurements is that the Higgs-Coulomb and the monopole percolation transitions are clearly distinct and, therefore, unrelated. This result will be confirmed on much larger lattices. This result stands in sharp contrast to fermion noncompact lattice QED and suggests that the physics of the phase transitions in the two 
models are quite different.

\section{FINITE SIZE SCALING AND THE SPECIFIC HEATS}

In order to understand the nature of the Higgs-Coulomb phase transition, we measured critical indices by doing a careful finite size scaling study of the specific heats related to the internal energies introduced above. We considered the specific heats $C_{\gamma}=\partial E_{\gamma} / \partial \beta$, and $C_{h}=\partial E_{h} / \partial \gamma$. In general, singular behavior in such specific heats at critical couplings can be used to find, classify, and measure the critical indices of phase transitions. On a $L^{4}$ lattice the size dependence of a generic specific heat at a second order critical point should scale as, ${ }^{10 .}$

$$
C_{\max }(L) \sim L^{\alpha / \nu}
$$

where $\alpha$ and $\nu$ are the usual specific heat and correlation length critical indices, respectively. Here $C_{\text {max }}$ denotes the peak of the specific heat. A measurement of the index $\nu$ can be made from the size dependence of the position of the peak. In a model which depends on just one coupling, call it $g$, then ${ }^{10 .}$

$$
g_{c}(L)-g_{c} \sim L^{-1 / \nu}
$$

where $g_{c}(L)$ is the coupling where $C_{\max }(L)$ occurs and $g_{c}$ is its $L \rightarrow \infty$ thermodynamic limit. The scaling laws Eq. (4) and (5) characterize a critical point with powerlaw singularities. This is a possible behavior for scalar electrodynamics, but there is also the possibility

suggested by perturbation theory, that the theory is logarithmically trivial. Consider $\lambda \phi^{4}$ as the simplest, well-studied theory which apparently has this behavior. In this case the theory becomes trivial at a logarithmic rate as the theory's momentum space cutoff $\Lambda$ is taken to infinity. Then the scaling laws of Eq. (4) and (5) become, ${ }^{11,12}$

$$
C_{\max }(L) \sim(\ln L)^{p}
$$


and

$$
g_{c}(L)-g_{c} \sim \frac{1}{L^{2}(\ln L)^{q}}
$$

where $p$ and $q$ are powers predictable in one-loop perturbation theory $\left(p=\frac{1}{3}\right.$ and $q=\frac{1}{6}$ in $\left.\lambda \phi^{4}\right)$. Note the differences between these scaling laws and those of the usual Gaussian model, obtained from Eq. (4) and (5) setting $\alpha=0$ and $\nu=.5$ : in the Gaussian model the specific heat should saturate as $L$ grows, and the position of the peaks should approach a limiting value at a rate $L^{-2}$.

It is particularly interesting in scalar electrodynamics to consider a large value of the bare (lattice) gauge coupling to see if that can induce non-trivial interactions which survive in the continuum limit. So, we ran extensive simulations on lattices ranging from $6^{4}$ through $20^{4}$ at $e^{2}=5.0$ and searched in parameter space $(\beta, \gamma)$ for peaks in $C_{\gamma}$ and $C_{h}$. We used histogram methods ${ }^{13,14}$ to do this as efficiently as possible. For example, on a $6^{4}$ lattice at $\beta=.2000$ and $\gamma=.2350$ we found a specific heat peak near $\gamma_{c}(6) \approx .2382$ from the histogram method. The peak is shown in Fig. 7. The $\gamma$ value in the lattice action was then tuned to .2382 and additional simulations and histograms produced specific heats, found from the variances of $E_{\gamma}$ and $E_{h}$ measurements, at a $\gamma_{c}$ very close to .2382. Using this strategy, measurements of $\gamma_{c}(L), C_{\gamma}(L)$ and $C_{h}(L)$ could be made without relying on any extrapolation methods. We thus avoided systematic errors, although critical slowing down on the larger lattices limited our statistical accuracy. In Fig. 8 and 9 we show the internal energy $E_{h}$ and specific heat $C_{h}$ on $12^{4}$ and $18^{4}$ lattices, respectively. Note that the peaks sharpen and shift to smaller $\gamma$ values as $L$ increases. These effects will be studied more systematically below when the shapes of each specific heat curve will be used to detect logarithmic scale breaking. In Table 1 we show a subset of our results that will be analyzed and discussed here. The columns labeled $\gamma_{c}(L), C_{\gamma}^{\max }(L)$ and $C_{h}^{\max }(L)$ in Table 1 need no further explanation except to note that the error bars were obtained with standard binning procedures which account for the correlations in the data sets produced by Monte Carlo programs. 
The Monte Carlo procedure used here was a standard multi-hit Metropolis for the noncompact gauge degrees of freedom and an over-relaxed plus Metropolis algorithm ${ }^{15}$. for the compact matter field. Over-relaxation reduced the correlation times in the algorithm by typically a factor of 2-3. Accuracy and good estimates of error bars are essential in a quantitative study such as this. Unfortunately, cluster and acceleration algorithms have not been developed for gauge theories, so very high statistics of our over-relaxed Metropolis algorithm were essential - tens of millions of sweeps were accumulated for each lattice size as listed in column 7 of Table 1 . We also wrote a unitary gauge code which eliminated the matter field entirely from the algorithm. Extensive runs on lattices ranging from $4^{4}$ to $16^{4}$ produced the same observables as the original code. These results provided an excellent check on the correctness of our programming and confirmed that our codes properly converged to statistical equilibrium.

A word of warning for the ambitious - the standard CRAY random number generator RANF which uses the linear congruent algorithm with modulus $2^{48}$ proved inadequate for lattices whose linear dimension was a power of 2 , such as $16^{4}$. Presumably this occurred because for strides of length $2^{N}$ the period of RANF is reduced from $2^{46}$ to $2^{(46-N)}$ and the well-known correlations in such generators are expected to have maximum effect if the distribution is sampled with a period of $2^{N}$. The simplicity of the variables and Monte Carlo algorithm for lattice scalar electrodynamics also makes it more susceptible to the correlations in random number generators than other models. We discovered this problem when our $16^{4}$ simulations were unstable - very long runs produced specific heat peaks that grew without apparent bound and shifted to large $\gamma$. After considerable investigative work, we isolated the problem in the random number generator. We cured the problem by adding extra calls to RANF to avoid strides of length $2^{N}$. Problems with generally accepted random number generators have been studied systematically in ref. (16)

Specific heats were measured as the fluctuations in internal energy measurements $\left(C_{h}=\right.$ $\left(<E_{h}^{2}>-<E_{h}>^{2}\right) / 4 L^{4}$, etc.). Very high statistics and many $L$ values are needed to distinguish between logarithmic triviality (Eq. 6) and powerlaw behavior (Eq. 4). The 
other entries in Table $1, K_{\gamma}(L)$ and $K_{h}(L)$, are the Binder Cumulants (Kurtosis) ${ }^{17}$. for each internal energy. At a continuous phase transition each Kurtosis should approach 2/3 with finite size corrections scaling as $1 / L^{4}$. The Kurtosis is a useful probe into the order of a phase transition, although an examination of the internal energy and specific heat histograms are often just as valuable. Since the order of the transitions in lattice and continuum scalar electrodynamics are controversial, we studied these quantities with some care.

Consider the Kurtosis $K_{\gamma}(L)$, the specific heat $C_{\gamma}^{\max }(L)$ and the critical coupling $\gamma_{c}(L)$ of scalar electrodynamics. As stated above, we set the lattice (bare) gauge coupling to $e^{2}=5.0$ and then used simulations, enhanced by histogram methods, to locate the transition line in Fig. 1. The Kurtosis $K_{\gamma}(L)$ is plotted against $10^{6} / L^{4}$ in Fig. 10. The size of the symbols include the error bars, and clearly the curve favors a second order transition. A three parameter fit to the $L=12,14,16,18$ and 20 data using the form $K_{\gamma}(L)=a L^{\rho}+b$ is excellent (confidence level $=98 \%$ ) predicting $\rho=-4.1(4)$ and $K_{p}(\infty)=.666665(2)$. The hypothesis of a line of second order transitions in Fig. 1 appears to be very firm, with no evidence for a fluctuation-induced first order transition. An analysis of $K_{h}(L)$ gives the same conclusion with somewhat larger error bars. In Fig. 11 we show $K_{h}(L)$ and find compatibility with the value $2 / 3$ for large $L$.

Next we plot our $C_{\gamma}^{\max }(L)$ data vs. $L$ in Fig. 12. We attempted powerlaw as well as logarithmic finite size scaling hypotheses. The powerlaw hypothesis did not produce a stable fit for any reasonable range of parameters. However, logarithmic fits were quite good. The hypothesis $C_{\gamma}^{\max }(L)=a \ell n^{\rho} L+b$ for $L=8,10,12,14,16,18$ and 20 fit with a confidence level $=90 \%$ producing the estimate $\rho=1.4(2)$. If we considered the range $L=8-18$, the same fitting form predicted $\rho=1.5(3)$ with confidence level $=84 \%$, and if the range $L=10-20$ were taken we found $\rho=1.4(5)$ with confidence level $=78 \%$. The solid line in Fig. 12 is the $L=8-20$ fit. An analysis of $C_{h}^{\max }(L)$ gave consistent results - the same logarithmic dependence should be found in either specific heat - and powerlaw fits to $C_{h}^{\max }(L)$ were also ruled out. In particular, a fit of the form $C_{h}^{\max }(L)=a \ell n^{\rho} L+b$ for $L=8-18$ gave $\rho=0.9(3)$ with confidence level $=82 \%$ and for $L=8-20$ gave $\rho=1.0(2)$ with confidence level $=85 \%$. 
We show the data and the logarithmic fit in Fig. 13.

Next, in Fig. 14 we show $\gamma_{c}(L)$ vs. $10^{4} / L^{2}$. As $L$ increases the specific heat peak shifts to smaller $\gamma_{c}(L)$, and the rate of the shift is determined by the critical index $\nu$ in a scaling theory. The error bars again fall within the symbols in the figure. The data is clearly compatible with the correlation length index $\nu=0.5$ expected of a theory which is free in the continuum limit. In the case of $\lambda \phi^{4}$ it has proven possible to find the logarithm of Eq. (7) under the dominant $L^{-2}$ behavior by using special techniques. ${ }^{18}$. We do not quite have the accuracy to do that here: a powerlaw fit to $\gamma_{c}(L)=\gamma_{c}+a / L^{1 / \nu}$ using $L=12-20$ predicts $1 / \nu=2.0(1), \gamma_{c}=.22825(8)$ with confidence level $=92 \%$ and using $L=14-20$ predicts $1 / \nu=1.9(3), \gamma_{c}=.2282(2)$ with confidence level $=97 \%$.

Taken together, these measurements of the size dependence of the critical couplings and specific heat peaks provide good evidence that SQED is logarithmically trivial. The measurement of $\nu$ is essential here - taken on its own, the specific heat data on the heights of the peaks would be less persuasive since one can cite models in less than four dimensions with nonzero anomalous dimensions but with just logarithmically singular or even nonsingular specific heats. However, in four dimensions hyperscaling correlates the powers $\nu=1 / 2$ and $\alpha=0$, and, on the basis of explicit calculations in $\lambda \phi^{4}$, the modifications of such scaling laws due to logarithms are rather well understood ${ }^{18}$. From this point of view, our measurements of $\nu$ and $\alpha$ are nicely consistent, and suggest that SQED may be logarithmically trivial in a fashion qualitatively similar to $\lambda \phi^{4}$.

Another insight into the dynamics of the model follows from the shapes of the specific heat peaks when plotted against the theory's bare couplings. As listed in Table 2 we did additional simulations at various $\gamma$ values on $12^{4}, 16^{4}$ and $20^{4}$ lattices to obtain the shapes of the specific heat $C_{h}(\gamma)$ for $\beta$ fixed at .2000. The results are plotted in Fig. 15 where we see that the peaks move to smaller $\gamma_{c}(L)$ and become narrower as $L$ increases.

In order to get such accurate results we used the multi-histogram techniques of Ref. 14 to combine data from different $\gamma$ values. We found that the method was effective only for $\gamma$ values near the $\gamma$ value used in the simulation itself. In particular, 1.5 million sweeps of the 
$16^{4}$ lattice were made at each $\gamma$ value listed in Table 2. The data lists at several $\gamma$ values above and below the one in question $\left(\gamma_{o}\right.$, say) were used to obtain additional predictions for $C_{h}\left(\gamma_{o}\right)$ and reduce its uncertainty. The errors quoted in the table come from standard binning methods treating each estimate of $C_{h}\left(\gamma_{o}\right)$ as statistically independent. We found that only nearby values of $\gamma$ were useful in reducing the variances-estimates of $C_{h}\left(\gamma_{o}\right)$ coming from simulations at very different $\gamma$ values had too much scatter to help pinpoint the actual $C_{h}\left(\gamma_{o}\right)$ value. Additional statistics at each $\gamma$ run would certainly improve the utility of the multi-histogramming methods, as observed by many other authors studying a wide variety of models. The histogram method was quite successful here, nonetheless, and the statistical errors reported in Table 2 are smaller by a factor of 2-3 as compared to the raw data at each $\gamma$ value.

The first point we wish to investigate is whether the data of Fig. 15 can be understood from the perspective of finite size scaling. If the theory were described by power-law singularities, then the specific heat data should follow a universal curve, ${ }^{10 .}$

$$
C_{h}(\gamma, L) \sim L^{\alpha / \nu} f(\Delta t)
$$

where

$$
\Delta t=\left(\gamma-\gamma_{c}(L)\right) L^{1 / \nu}
$$

Clearly Eqs. (8a, b) generalize Eqs. (4) and (5) above. With scale breaking logarithms we expect instead $^{18}$,

$$
C_{h}(\gamma, L) \sim(\ln L)^{p} f(\Delta t)
$$

where

$$
\Delta t=\left(\gamma-\gamma_{c}(L)\right) L^{2}(\ln L)^{q}\left(\ln \left(\gamma-\gamma_{c}(L)\right)\right)^{r}
$$

In either case, Eq. (8) or Eq. (9), the specific heat peaks should increase with $L$ and become narrower when plotted against $\gamma$. These qualitative effects are clear in the data. Given data on just three lattice sizes the functional form of the scaling prefactors in Eqs. (8a) and (9a) 
will not be challenged here, but the widths of the peaks can provide some insight. We expect that Eq. (8b), with $\nu$ set to .50, will be fairly successful in describing the narrowing of each peak in light of Fig. 14. In Fig. 16 we plot the $12^{4}, 16^{4}$ and $20^{4}$ data in the form of Eq. (8) after rescaling the height of each peak to the $12^{4}$ data, using the more accurate data for the peaks in Table 1. The "near universal" character of the data is clear with $\nu=1 / 2$ but as $L$ increases the data falls systematically below Eq. (8). It is interesting, however, that the scaling form of the data can be markedly improved by including a logarithm of scale breaking as suggested by Eq. (9). In Fig. 17 we replot the data, scaled to a common height, using Eq. (9b) with $q=1$ and $r=0$. The curves in Fig. 17 overlap beautifully now, giving good evidence that logarithmic corrections to gaussian exponents can accommodate the entire data set.

We can find additional evidence for logarithmic violations of scaling and triviality by analyzing the $\gamma$ dependence of each peak. For infinite $L$ the specific heat should diverge logarithmically in this scenario,

$$
C_{h}(\gamma, L \rightarrow \infty) \sim \ln n^{p^{\prime}}\left|\gamma-\gamma_{c}\right|
$$

On a finite lattice this sort of result is, in general, hard to confirm because it depends on the existence of a "scaling window"-for each $L$ one must find a range of $\gamma$ where Eq. (10) holds, undistorted by finite size effects which occur when $\gamma$ is chosen too close to $\gamma_{c}$ and undistorted by finite lattice spacing effects which occur when $\gamma$ is chosen too far from $\gamma_{c}$. We considered the $16^{4}$ data and tried fits of the form $C_{h}(\gamma, 16)=a \ln ^{p^{\prime}}|\Delta \gamma|+$ b both above and below the peak. Choosing the points at $\gamma=.2300-.2310$ we found a fit with a $65 \%$ confidence level yielding only rough estimates of the parameters $a=1.26(2.91), p^{\prime}=1.39$ (.94) and $b=1.14$ (9.59). Fits of similar quality were found on the other side of the peak. Simple logarithmic plots are shown in Figs. 18 and 19 demonstrating consistency of the data with a weak logarithmic divergence. Clearly this "brute force" approach is not nearly as quantitative or decisive as the finite size scaling study of the peak heights, but it is certainly 
compatible with that data. It is interesting that power-law fits, $C_{h}(\gamma, 16)=a|\Delta \gamma|^{-\alpha}+$ b are not stable-the fitting procedure always finds it can reduce the chi-squared of a fit by reducing $\alpha$ while " $a$ " grows positively and " $b$ " grows negatively, thus approximating a logarithm.

\section{MONOPOLE PERCOLATION}

We made a detailed study of monopole percolation in the vicinity of the HiggsCoulomb transition studied quantitatively above. We used finite size scaling methods since they have been so succesful in similar studies done elsewhere. In particular, lattices ranging from $6^{4}$ through $24^{4}$ were simulated at the $\gamma_{c}(L)$ values determined from the specific heat peaks discussed above. The monopole order parameter $M$ and its associated susceptibility $\chi$ were then calculated over a range of $\beta$ values. The results of these simulations on $6^{4}, 12^{4}$, and $18^{4}$ lattices are shown in Fig. 20, 21, and 22, respectively. Typically, only 50,000-100,000 sweeps of the algorithm were needed to obtain this data with their relatively small error bars. We see from the figures that a very clear percolation transition appears at $\beta=.2325$. As was also found in our cruder simulations which mapped out the phase diagram, the monopole percolation transition is not coincident with the Higgs-Coulomb transition.

We can obtain several critical indices of the percolation transition by using scaling arguments. For example, the peak of the monopole susceptibility peak should depend on $L$ as,

$$
\chi_{\max } \sim L^{\gamma / \nu}
$$

where $\gamma$ and $\nu$ are the susceptibility and correlation length exponents. We test this scaling law in Fig. 23 where we see that the power law works very well with $\gamma / \nu=2.25(1)$. This result is in excellent agreement with the scaling law of ordinary four dimensional percolation, $9 / 4^{19}$. Since the bulk specific heats are not critical at this point, it is not surprising that the monopole percolation indices would be uneffected by the Higgs field. We attempted 
other measurements of critical indices at the percolation point, but they proved to be less quantitative.

\section{FOUR DIMENSIONAL PLANAR MODEL}

To check our results for the full theory, we confirmed that our techniques were able to reproduce known results. For example, when $\beta \rightarrow \infty$ Eq. (1) reduces to the four dimensional planar spin model which should have an order-disorder transition as a function of $\gamma$ that is described by mean field theory $(\alpha=0, \nu=.5$, etc. $)$ with logarithmic corrections calculable in perturbation theory ${ }^{20}$ - for example, the index $p^{\prime}$ in Eq.(10) is predicted to be $1 / 5$ for the $\mathrm{O}(2)$ model. We measured the specific heat at the transition for $L=6,8$, 10, 12 and 14, and found peak values 20.47(3), 22.80(5), 24.35(9), 25.38(9) and 26.24(9), respectively. One million sweeps of our code, tailored for $\beta=\infty$, were run in each case. We note that for $L>6$, the specific heat peaks grow with $L$ at a rate for which is almost identical to the specific heat peaks in the full theory. This numerical result is consistent with the perspective developed above - introducing the gauge coupling in the model does not change the theory qualitatively. The specific heat data are shown in Fig. 24. We also checked that the correlation length exponent $\nu$ for this limit of SQED is compatible with mean field theory. The peaks in the specific heat occurred at $\gamma=.1556(1)$ at $L=6$, $.1541(1)$ at $8, .1532(1)$ at $10, .1526(1)$ at 12 , and .1523(1) at 14. As shown in Fig. 25, these

measurements are perfectly compatible with the scaling law $\gamma_{c}(L)=a L^{-1 / \nu}+b$ and the mean field value $\nu=1 / 2$. As was the case in SQED, the data is not quite accurate enough to search for the logarithms of Eq.(7). And finally, in Fig. 26 we show the Kurtosis plot for the planar model and see that it is compatible with the value $2 / 3$ for large $L$. Certainly much more exacting studies of this model could be made (cluster algorithms) and much larger lattices could be simulated, but we are testing here just the simulation and analysis technology available to the gauge model. The success of this test study gives us confidence that our SQED conclusions are reliable and the logarithmic violations of mean field theory 
in SQED are real.

\section{CONCLUDING REMARKS}

One of the motivations for this study was the recent finding that the chiral symmetry breaking transition in non-compact lattice electrodynamics with dynamical fermions is consistent with an ultra-violet stable fixed point. ${ }^{3 .}$ Powerlaw critical behavior has been found with non-trivial critical indices satisfying hyperscaling. The present negative result for scalar electrodynamics suggests that the chiral nature of the transition for fermionic electrodynamics is an essential ingredient for its scaling behavior. It remains to be seen, however, if the chiral transition found in fermion noncompact lattice QED produces an interesting continuum field theory.

In conclusion, our numerical results support the notion that scalar electrodynamics is a logarithmically trivial theory. We suspect that this result could be made even firmer by additional simulation studies which use more sophisticated techniques such as renormalization group transformations ${ }^{5}$ or partition function methods. ${ }^{18 .}$ We are also hopeful that the data in Table 2. can be better organized and exploited than we did here, and the presence of logarithmic scaling violations can be extracted more quantitatively from this finite size study. Since we did not wish to bias our study toward logarithmic triviality, we did not pursue special methods which require additional theoretical input in order to be quantitative. However, it now seems appropriate to execute a study of this type. ${ }^{18 .}$ Certainly our concentration on a line of fixed electric charge in the entire phase diagram should be relaxed. Hopefully, accelerated Monte Carlo algorithms could be developed for scalar electrodynamics so that larger systems could be simulated with better control.

We have presented calculations and fits to simulation data on a wide range of lattice sizes and couplings, but since the logarithms of interest are so slowly varying, we are skeptical that our determinations of the exact powers of the various logarithms are very quantitative. In particular, we know from perturbative studies of $\lambda \phi^{4}$ that logarithmically divergent 
specific heat peaks are accompanied by additive corrections that fall away very slowly, as a small negative power of the logarithm. It would take a wider range of couplings and lattices to accommodate such nonleading terms meaningfully into our fits, and once that could be done, we suspect that the powers of the leading logarithmic singularities discussed here could change quite significantly. Greater analytic insight into SQED, or much more penetrating data analysis methods appear necessary to quantitatively determine the powers of the logarithms of interest with confidence. Nonetheless, we feel that the primary goal of this research project was achieved - SQED is compatible with logarithmic triviality and powerlaw critical behavior indicative of a nontrivial ultra-violet stable fixed point has no support.

\section{ACKNOWLEDGEMENT}

The simulation done here used the CRAY C90's at PSC and NERSC. We thank these centers for friendly user access. Several thousand cpu hours were needed to accumulate the statistics listed in Table 1. The authors thank D. K. Sinclair for help in the early stages of this work and acknowledge his assistance in solving the random number problems discussed in the text. J.B.K. is supported in part by the National Science Foundation grant NSF PHY9200148. S.K. is supprted by DOE contract W-31-109-ENG-38. M.B. and H.F. acknowledge the support of CESCA, CIEMAT and CICYT (project AEN \#93-0474). H.F. acknowledges support from CEE.

\section{REFERENCES}

1. M. Baig, H. Fort, J.Kogut, S. Kim, and D. K. Sinclair, Phys. Rev. D45, R2385 (1993).

2. L. D. Landau and I. Ya. Pomeranchuk, Dokl. Akad. Nauk. 102, 489 (1955). 
3. S. Hands, A. Kocic, J. Kogut, R. Renken, D. K. Sinclair, and K. C. Wang, Nucl. Phys. B413, 503 (1994).

4. M. Baig, E. Dagotto, J. Kogut and A. Moreo, Phys. Lett. B242, 444 (1990).

5. D. Callaway and R. Petronzio, Nucl. Phys. 277B, 50 (1980).

6. J. L. Alonso et al., Zaragoza preprint, Sep 25, 1992.

7. C. N. Leung, S. T. Love, and W. A. Bardeen, Nucl. Phys. B323, 493 (1989).

8. S. Hands, and R. Wensley, Phys. Rev. Lett. 63, 2169 (1989).

9. T. Banks, R. Meyerson, and J. Kogut, Nucl. Phys. B129, 493 (1977). T. A. DeGrand and D. Toussaint, Phys. Rev. D22, 2478 (1980).

10. M. N. Barber, in Phase Transitions and Critical Phenomena, Vol. VIII, eds. C. Domb and J. Lebowitz (Academic Press, New York: 1983).

11. E. Brezin, J. Physique 43, 15 (1982).

12. J. Rudnick, H. Guo and D. Jasnow, J. Stat. Phys. 41353 (1985).

13. M. Falcioni, E. Marinari, M. L. Paciello, G. Parisi and B. Taglienti, Phys. Lett. 108B 331 (1982).

14. A. M. Ferrenberg and R. H. Swendsen, Phys. Rev. Lett. 61, 2635 (1988).

15. M. Creutz, Phys. Rev. D36, 515 (1987).

16. A. M. Ferrenberg, D. Landau and Y. J. Wong, Phys. Rev. Lett. 69, 3382 (1992).

17. K. Binder, M. Challa and D. Landau, Phys. Rev. B34, 1841 (1986).

18. R. Kenna and C. B. Lang, Phys. Lett. 264B, 396 (1991).

19. S. Hands, A. Kocic and J. Kogut, Phys. Lett. 289B, 400 (1992).

20. E. Brezin, J. C. Le Guillou and J. Zinn-Justin, Phys. Rev. B8, 2418 (1973). 


\section{FIGURE CAPTIONS}

1. The phase diagram of non-compact scalar electrodynamics.

2. A $\beta=0.1$ scan of the internal energies.

3. Same as Fig. 2, except at $\beta=0.2$.

4. Contour plot of $E_{\gamma}$.

5. Same as Fig. 4, except $E_{h}$.

6. Phase diagram on $6^{4}$ lattice showing line of specific heat peaks and line of monopole susceptibility peaks (dashed).

7. Specific heat histogram on a $6^{4}$ lattice.

8. Same as Fig. 8, except on $12^{4}$ lattice.

9. Same as Fig. 9, except on $18^{4}$ lattice.

10. The Kurtosis $K_{\gamma}(L)$ vs. $10^{6} / L^{4}$.

11. Same as Fig. 11, except for $K_{h}$.

12. The specific heat peaks $C_{\gamma}^{\max }(L)$ vs. $L$. The solid line is the logarithmic fit discussed in the text.

13. Same as Fig. 13., except for $C_{h}^{\max }(L)$.

14. The critical coupling $\gamma_{c}(L)$ vs. $L^{-2}$.

15. Specific heat peaks vs. $\gamma$ on $12^{4}, 16^{4}, 20^{4}$ lattices.

16. Universal specific heat plot for powerlaw scaling.

17. Same as Fig. 17 except with scale breaking logarithm. 
18. Coupling constant dependence of specific heat on $16^{4}$ lattice, $\gamma<\gamma_{c}$ (16).

19. Same as Fig. 19, except $\gamma<\gamma_{c}$ (16).

20. The monopole susceptibility and order parameter plotted vs. $\beta$ on a $6^{4}$ lattice.

21. Same as Fig. 16., except on a $12^{4}$ lattice.

22. Same as Fig. 16., except on a $18^{4}$ lattice.

23. Plot of $\ln \chi_{\max }$ vs. $\ln L$.

24. Plot of $\ln C_{\max }$ vs. $\ln L$, in the planar model.

25. Plot of $\gamma_{c}(L)$ vs. $1 . / L^{2}$, in the planar model.

26. Kurtosis plot for the planar model. 


\section{TABLES}

TABLE I. Finite Size Study of Scalar Electrodynamics

\begin{tabular}{lllllll}
\hline \hline $\mathrm{L}$ & $\gamma_{c}(L)$ & $C_{h}^{\max }(L)$ & $K_{h}(L)$ & $C_{\gamma}^{\max }(L)$ & $K_{\gamma}(L)$ & Sweeps(millions) \\
6 & $.23815(1)$ & $13.81(2)$ & $.657668(9)$ & $7.965(9)$ & $.665784(2)$ & 40 \\
8 & $.23375(3)$ & $15.83(2)$ & $.662954(5)$ & $8.083(3)$ & $.666374(1)$ & 60 \\
10 &. $.23173(1)$ & $17.23(4)$ & $.664892(4)$ & $8.285(6)$ & $.666544(1)$ & 60 \\
12 & $.23070(1)$ & $18.43(7)$ & $.665713(4)$ & $8.457(9)$ & $.666606(1)$ & 30 \\
14 & $.23004(1)$ & $19.38(9)$ & $.666110(3)$ & $8.594(15)$ & $.666633(1)$ & 20 \\
16 & $.22962(1)$ & $20.25(13)$ & $.666319(2)$ & $8.747(17)$ & $.666647(1)$ & 12 \\
18 & $.22933(1)$ & $20.85(15)$ & $.666441(2)$ & $8.863(26)$ & $.666654(1)$ & 12 \\
20 & $.22912(1)$ & $21.76(20)$ & $.666510(2)$ & $8.956(20)$ & $.666658(1)$ & 10 \\
\hline \hline
\end{tabular}


TABLE II. Specific Heat Data on $12^{4}, 16^{4}, 20^{4}$ Lattices

\begin{tabular}{|c|c|c|c|}
\hline$\gamma$ & $C_{h}(12)$ & $C_{h}(16)$ & $C_{h}(20)$ \\
\hline .2278 & - & - & $5.58(8)$ \\
\hline .2280 & - & - & $5.99(6)$ \\
\hline .2282 & - & - & $6.88(7)$ \\
\hline .2284 & - & $7.68(7)$ & $8.32(11)$ \\
\hline .2286 & - & $9.01(8)$ & $11.25(19)$ \\
\hline .2288 & - & $10.96(9)$ & $16.31(24)$ \\
\hline .2290 & - & $13.53(13)$ & $20.54(29)$ \\
\hline .2292 & - & $16.42(16)$ & $21.25(40)$ \\
\hline .2294 & $11.56(5)$ & $19.07(16)$ & $18.51(29)$ \\
\hline .2296 & $12.95(5)$ & $20.07(46)$ & $16.36(14)$ \\
\hline .2298 & $14.41(5)$ & $19.49(20)$ & $15.16(13)$ \\
\hline .2300 & $15.82(5)$ & $17.90(15)$ & $14.30(22)$ \\
\hline .2302 & $17.03(5)$ & $16.48(9)$ & - \\
\hline .2304 & $17.88(6)$ & $15.29(10)$ & - \\
\hline .2306 & $18.27(8)$ & $14.57(8)$ & - \\
\hline .2308 & $18.18(14)$ & $13.99(8)$ & - \\
\hline .2310 & $17.99(16)$ & $13.79(12)$ & - \\
\hline .2312 & $17.31(14)$ & - & - \\
\hline .2314 & $16.52(11)$ & - & - \\
\hline .2316 & $15.72(9)$ & - & - \\
\hline .2318 & $14.91(8)$ & - & - \\
\hline .2320 & $14.31(7)$ & - & - \\
\hline
\end{tabular}

